\title{
Operating System Support for Multimedia
}

\author{
Praveen Moorthy \\ Motorola Broadband Communications Sector, \\ 6450 Sequence Drive, San Diego, CA - 92122 \\ praveen.moorthy@motorola.com
}

\section{Introduction}

The last couple of years have seen an increase in multimedia content in almost every context web pages (MPEG clips, Flash animations), desktop (MediaPlayer, QuickTime, NetMeeting), consumer products (DVD players, Set-top Boxes, Interactive TV). These content-rich applications rely on extensive support by the operating system (OS). The users' thresholds of tolerance for performance degradation vary largely on the type of content - delay/jitter in static text and images is acceptable, dropped frames or lower resolution in video is moderately acceptable whereas even the slightest degradation in audio (in the form of scratch, skip or pop) is not acceptable at all. To add to this, further complications arise in user desktops where a mix of applications is run simultaneously with different levels of performance degradation. Therefore, good OS support is very essential for multimedia applications.

\section{Characteristics of Multimedia}

Most of the multimedia contexts existing these days have very characteristic parameters. This characterization leads to a more customized design per the needs and wants of the applications displaying the content and better resource utilization. Digital audio needs a fixed sampling of $44 \mathrm{kHz}$ while video MPEG rendering at $30 \mathrm{fps}$ is desirable. The processing need for both these types is periodic and sequential in nature. However, MPEG frames can be either IFrames (computation intensive) or B/P Frames (difference information).

This behavior leads us to the conclusion that multimedia applications need real-time support from the underlying OS. This requirement is flexible as missing of a certain deadline does not lead to a catastrophic failure. Either the information that was being processed is just discarded (B/P Frame) or the computation is allowed to continue for a short duration, if it has important information for subsequent computations (I-Frame). Additionally, users would prefer a consistent low frame rate or resolution to a randomly varying rate or resolution with an average close to 30 FPS.

Thus, multimedia applications need the support of soft real-time OS's to be able to guarantee a certain level of QoS.

\section{General Purpose OS for Multimedia}

Multimedia applications on the desktop computers rely on the support provided by the general purpose OS's - flavors of UNIX (Solaris, HP Unix, Linux, OS-X) and flavors of Windows. In an attempt to match the requirements imposed by multimedia, these OS's have been refurbished in key areas. They provide an extra class of priority called real-time priority. Multimedia applications can be made to run at this level to achieve some of its requirements. The experimental results of using SRV4 Unix are described in [2]. The experiments consisted of comparative analysis when three classes of applications - interactive, multimedia rich and batch applications were run simultaneously on the workstation. The results clearly indicate that the multimedia applications can no doubt benefit from the real-time priority class but at the cost of degraded performance of both the interactive and batch applications. Additionally, this also affects the system services running as demons, which run at high priority (not real-time), to a large extent.

The hardware these days consist of fast processors, large and comparatively fast primary and secondary storage and good network bandwidth. However, multimedia applications perform poorly. This is clearly indicated by the small window size of the video players on the desktops. Besides this, the OS's do not support mechanisms to ensure guaranteed completion of a task before a certain deadline. To add to this, kernel operations such as page fault handling, cache miss, interrupts from I/O devices, critical sections and context switch overhead cause unpredictable runtime behavior. The key to good 
QoS therefore boils down to good resource allocation policies provided by the OS.

\section{Resource Allocation}

Applications often view the OS as an abstraction of the hardware, which comprises of different resources - CPU, memory, disk and I/O devices. In order to arbitrate various applications from over usage and contention, policies and mechanisms are required for efficient resource utilization. A consolidated list of areas is discussed in [1].

\subsection{CPU Scheduling}

This is one of the key issues, which contribute to the QoS of the system. Extensive research is being carried out in this area, especially for multimedia systems.

\subsubsection{Static Priority Scheduling RTOS's}

Static priority scheduling is the traditional approach taken by most embedded/real-time OS's like VxWorks, pSoS, VRTX etc. This approach is good only if one or few applications run to completion or if the complete nature of the system is known a priori. The relative priorities of all the tasks in the system are decided during system design, hence it is static and not dynamic. This may not be easily determinable as it is difficult to map application timing and constraints into priority levels. This priority based preemptive scheduling imposes constraints on the applications but does not force any constraints on resources. Also, misbehaving high priority applications can affect well-behaving low priority applications. Additionally, RTOS's are characterized by very low interrupt latency. This can be an advantage but it also leads to uncertainty in the system and could lead to lost deadlines.

\subsubsection{Earliest Deadline First}

Earliest Deadline First is a very old and proven form of scheduling, commonly used for hard real-time task scheduling. It is a dynamic scheduling policy wherein the deadlines of all tasks are re-calculated and re-adjusted whenever a task is added or removed from the set of executable tasks. The tasks under consideration are usually periodic tasks, with fixed start times and an associated period. The system calculates the window, from start time to a finish time within which the task will be scheduled. This no doubt provides guarantees, but it also forces applications to be ready to use the resource at any point in the window. This may be inappropriate if a task needs to do computations based on I/O data, for which it needs to block for a very short duration. This may lead to starvation, in some cases. As suggested by the name, the task with the earliest deadline is always executed first. This type of scheduling provides good performance for under-load and optimal load conditions but unpredictable performance under overloads.

\subsubsection{Lottery Scheduling}

Judging by the name "Lottery" this scheme appears to be unsuitable for real-time applications due to its inherent random nature. However, [3] discusses the design of such a scheduler for the Mach 3.0 micro-kernel and its performance on different classes of applications including multimedia.

The basic mechanism is to allow applications to hold on to lottery tickets of possibly variable currency. The scheduler conducts lotteries and the winner is scheduled. The throughput of a task is directly proportional to the number of tickets it holds and the response time is inversely proportional to it i.e. the more the number of tickets a task holds, the lesser it has to wait. This is a very fair proportional share scheduling policy and due to this there is no starvation of any low priority tasks. The scheme of ticket transfer allows a client to transfer its ticket to the server to increase its response time, and take the ticket back after the call. A task can distribute its tickets among its threads and control the behavior by inflating or deflating the value of each ticket.

It also provides an interesting system-controlled mechanism called compensation tickets. A thread consuming only a fraction of its allocated resources is automatically allocated a temporary, proportional compensation ticket to boost its computation. The major benefit of this scheduling mechanism is its lightweight algorithm, thereby reducing the computation overhead in the kernel. Their experiments on multimedia show the ease of use and its good responsiveness. Additionally, situations where low priority applications starting before a high priority applications grabbing more tickets can be easily handled by dynamically adjusting the value of the tickets. The main drawback of this scheme is that tasks can only assume their rate of scheduling using probability - this may not represent the actual behavior at runtime. Of course, the random number generator used is 
actually pseudo-random hence patterns can be established but is not recommended as the algorithm may change over time.

\subsubsection{Hierarchical Start-Time Fair Scheduling} This is a very interesting scheduling algorithm. It consists of a hierarchical tree of schedulers each for specific classes of applications; i.e. the nodes of the tree are different schedulers themselves. The leaves represent the different tasks in the system. When a task needs to be scheduled, the root selects one of the nodes according to SFQ (described in the next paragraph). This node in turn governs the scheduling policy of its nodes. This node traversal continues until the task to be schedules on a leaf node is reached. This deviation from the general purpose OS approach, where all applications are scheduled according to one fixed policy, provides good protection and insulation among the groups. This approach has many advantages as the scheduling needs of applications are very different - some are interactive (need low response time), some are batch (computation intensive) and some are time bound (need guaranteed resource allocation and completion within a deadline). Due to this tree structure, addition of new schedulers and changing of the scheduling policy of a task is trivial and can be accomplished dynamically.

The main scheduling policy used for deciding which node to select from the root node is based on Start-time Fair Queuing. As per this scheme, start times are dynamically attached to tasks each time they are preempted. This start time is calculated based on their individual weights so that the CPU share received is proportional to the weight. This weight of each task can also be changed dynamically. Despite the scheduling mechanism being hierarchical in nature, the associated overhead is comparatively low $\sim 1 \%$ as per their results. This is really low compared to the extent of flexibility achieved. Unlike other real-time schedulers for multimedia, it does not require the specification of a deadline during task creation. The framework calculates the start time and schedules the task as per its weight. The allocation of weights is relative in nature; i.e. when new tasks get added the individual weight does not change but the proportional allocation changes as per the equation $\frac{w_{i}}{\sum_{j} w_{j}}$ where $w_{i}$ is the weight of task $i$. This ensures good scheduling despite overload.

\subsubsection{SMART}

Scheduler for Multimedia and Real-Time Applications [4] is a scheduler designed to replace the conventional scheduler in Solaris. The motivation for this is the poor performance of the existing scheduler as indicated in [2]. The focus of the design is to schedule and make loadshedding decisions based on user preferences. Users may prefer degraded video to timely completion of their code compilation. This is a very good approach as the decision making is not static and changes not only per the runtime environment but also per the users requirements. SMART uses the notation of importance and urgency. Basically, they refer to priority and deadline as described in other schedulers.

The programming model expects time constraints (consisting of estimates and deadlines) and a mechanism for overload notification using up-calls, to be provided at task creation. The user can also change the behavior dynamically by changing the priority and providing a value for proportional sharing of resources in case of contention. Tasks are kept in different queues based on their priority. Each task is assigned a dynamically changing virtual finish time - VFT. To this, a certain amount of bias is added, proportional to its tolerance level for delay - leading to a BVFT. A two-pass approach is taken to select a task set for execution. In the first pass, the importance is considered (tasks with higher priority). In the second pass, this set is reordered based on the lowest value of BVFT (earliest deadline first as described in 4.1.2). After this, a feasibility test is done to check if the task set can proceed to execution without missing a deadline. This entire process is done iteratively, until all tasks have completed their execution.

This is a very complex scheduling mechanism and with increase in number of tasks, queue traversal can prove to be expensive. Also, due to their two-pass approach, a time-sharing task with higher priority will be selected before a real-time task about to miss a deadline. This is acceptable as it is in line with their goal. If a user wishes to overcome such a situation, it can be achieved easily by dynamically changing the priority of the real-time task. To prove their design, they have conducted extensive tests and from the 
results it is evident that their design is indeed very smart!

\subsection{Resource Distributor}

The Resource Distributor [5] takes an aggressive approach to resource allocation and distribution for multimedia applications. Unlike other models, it provides hard real-time guarantees. The framework requires that applications provide various load shedding options corresponding to various QoS levels during task creation. Each level corresponds to different levels of resource grants over a period of time. Task creation goes through a strict admission control policy, which allows it if and only if the sum of the minimum grants of all existing tasks including that of the new task is $\leq 100 \%$. This process involves consultation of a Policy Box, in case of conflicts. This ensures global QoS decisions. The cost of consultation, however, is imposed on the task requesting admittance. Once a task is admitted, it is guaranteed not to miss a deadline. Additionally, nearly $100 \%$ of available resources is always allocated to the ready task. Any available slack time from other processes is also completely provided to it. This makes sure that resource utilization is kept as high as possible. All the admitted tasks are scheduled using an EDF scheduler as mentioned in 4.1.2.

The EDF scheduler fits well into their framework and is comparatively light. Additionally, they have provided a certain amount of flexibility in the system such that if the overhead due to a context switch is more than completion time of the current task then the task is allowed to complete. The limitation imposed by EDF, requiring tasks to be periodic is overcome using a sporadic server. This sporadic server schedules all non-periodic tasks using round robin. Due to this, no guarantees are provided for the timely completion of any of these tasks. The sporadic server, itself being a task in the system is scheduled using EDF.

The admission control mechanism may prevent spawning of some important kernel operations during system overload. To prevent this, the system provides the notion of quiescent tasks. These predefined kernel tasks are used in the calculations during admission control but no resources are granted to them until they actually get spawned. The system handles overload situations very gracefully by notifying the concerned tasks to shed load. This provides a clear separation between scheduling and QoS decision-making. The framework has high associated overheads due to admission control, grant set calculation etc but the benefits achieved are manifold. Also, from their experiments, these overheads are in the range of about $100 \mu \mathrm{s}$, which I think is acceptable.

\subsection{Disk and Memory Usage}

Multimedia applications tend to work on large amount of real-time data (for example: live video feed coming form a camera or a video server providing video on demand). In such situations, disk I/O can be highly time bound. However due to the nature of this data, a lot of algorithms used for CPU scheduling can also be applied to disk scheduling. The main goal is to provide reduced seek times and optimizations for continuous data stream. [1] discusses the different schemes present for disk scheduling and memory management. Rather than considering them as different issues, I feel that they need to be addressed together.

Pre-fetching from disk, based on application level hints is very beneficial due to the nature of multimedia data. Additionally, due to the nature of the data usage, redundant copying from disk to memory, memory to memory, memory to cache and memory to network occur. Other multimedia specific behaviors such as stream merging and content insertion are also memory intensive. Using various VM techniques all these issues can be handled together and optimized. Cache, on the other hand adds unpredictability to multimedia applications due to inherently large sizes of the data. [1] suggests some issues such as partitioning the L2 cache into two areas, one for time-sharing and the other for real-time. Overall, primary and secondary storage need to work in harmony to provide good bandwidth and throughput

\subsection{Intelligent Network Interface}

In the attempt to optimize and pipeline the computations for multimedia application entering the system via the network, [6] takes the approach of adding more intelligence in the NIC. This reduces a certain amount of burden from the CPU and removes redundant copying by directly writing to the frame buffer. The motivation for this approach is to reduce the widening gap between the raw CPU processing power and the speed of the I/O device in the system. Applications can be written in a type safe language and downloaded into a runtime environment called SPINE. This runtime 
environment consists of two parts - one provided in the NIC card for I/O and another in the kernel. This allows an isolated environment for downloading applications and usage of both I/O environment and calls to the kernel on the main CPU.

Communication is via active messages messages contain data and control information. The SPINE environment acts as the resource manager for the application and provides safe access to the underlying processor for computations and DMA for transferring the data from the NIC into the buffer. The results form their experiments (40 MBPS sustained data rate and CPU utilization $0 \%$ ) clearly indicate that is framework removes a major part of the network based multimedia burden from the CPU by handling the network packets and MPEG decoding. Thus, any general purpose OS with minor modifications can be used to run on the main CPU. Although this approach focuses on the NIC, I feel that this can be extended to other I/O bound areas by providing similar accelerator cards using cheap and easily available microprocessors.

\section{Case Studies}

The following subsection discusses three interesting OS's designed to support multimedia. Although there are numerous other research papers, I selected these as they had a lot of useful and distinct approaches towards a common goal.

\subsection{YARTOS}

YARTOS [7] is a micro-kernel developed by the CS department of University of North Carolina for research on multimedia applications. It imposes a certain programming model on application developers so that applications can make use of the underlying OS architecture for ensuring real-time guarantees. It uses message passing as the basic form of IPC with real-time delivery guaranteed by the OS. It, however, requires tasks to be sporadic in nature. This is not a very serious requirement as multimedia applications perform computations periodically on the packets of data they receive. The system attempts to sequence all the tasks such that, as far as possible, all can meet their deadlines. Also, they are all made to run in a single stack without any explicit locking mechanism. This reduces a lot of the overhead and allows effective data sharing among tasks.
Tasks $\mathrm{T}$ are defined as $\mathrm{T}=(\mathrm{C}, \mathrm{R})$ with $\mathrm{a}$ computational cost $\mathrm{C}$ representing the processing time needed for the task to complete and a response time $\mathrm{R}$ representing the minimum time interval between successive invocations. The scheduling mechanism used is basically EDF as described in 4.1.2. However, in their model, tasks are sequentially ordered and no resource locks are used. Hence, when a task accesses a shared resource, its deadline is automatically shifted by a value representing the time when the resource will be accessible without causing contention. This mechanism effectively provides mutual exclusion between different tasks that need to access the same resource. It also has a mechanism for admission control with which a request for spawning a task is checked if it can be executed without causing any deadlines to be missed. All interrupts are converted into tasks and scheduled along with other tasks. This definitely makes the interrupt latency unpredictable but it ensures that interrupts do not cause unreliability in the executing tasks.

The experimental results show that the programming model is very light. It has been implemented as a TSR program on DOS. The multimedia applications they have tested are virtual reality and video conferencing. Both these are computation intensive multimedia applications. Overall, their programming model is effective. However, it needs the tasks to know their precise computational and resource requirements. This is not a very heavy imposition as multimedia applications have a very characteristic behavior (as explained in 2).

\subsection{Rialto}

Rialto [8] is a real-time OS written by the research group at Microsoft. It is designed to support dynamic scheduling of tasks based on the set of constraints provided by the application. Applications can put timing constraints by calling BeginConstraint(start-time, estimate, deadline, criticality), where start-time is the earliest time to start execution of the code, estimate time is the estimated time for the code execution, deadline is the time within which the code must be executed and criticality is a provision for hard real-time behavior. The call does an admission control check and returns a value indicating whether it can be scheduled or not. This is useful feedback information for applications to gracefully shed load, if possible. 
The end of the constraint is indicated by calling the EndConstraint(). This call returns the actual time that was spent executing the code above i.e. it is not the elapsed time since the code started and finished, but the amount of time that was spent on the CPU. This is very useful information, and can be used as feedback for subsequent calls as the estimate parameter of BeginConstraint(). This eliminates the need for the application developer to make very fine grain calculations about the time of the code., which may be different on different pieces of hardware. Timing constraints get automatically transferred from a client to a server reducing the chance of a missed deadline during a RPC.

The resource planner does resource management at a global level. Resource objects register themselves with the resource manager and applications gain access to these resources via the resource manager. The resource manager does the arbitration and re-negotiation of resources among various applications. Applications are represented as activity objects consisting of threads. Only activities are allowed to reserve resources and the accounting of resources is done per activity and not per thread. Scheduling is done as per the CPU resources reserved by the activity. Within the threads of an activity, fair share scheduling is done as far as possible and preference is given to threads with closer deadlines. In order to prevent starvation, a very simple approach is used. $5 \%$ of $\mathrm{CPU}$ is always kept unreserved. All the unreserved time is evenly shared between all activities. This could have been extended by allowing a dynamically changing value so that this unreserved time could be made use of on the occurrence of an overload.

The experimental results clearly indicate the effectiveness of their system. Their model is simple to use, however the added overhead due to the resource manager is not discussed. Additionally, complexity of resource accounting occurs due to kernel calls (interrupts, context switch time etc.), which should not be accounted to the application that is scheduled when these occur. This fine level of accounting is also not discussed in the paper.

\subsection{QLinux}

QLinux [9], designed by the CS department of University of Massachusetts, takes the approach of modifying key components of an existing, stable, desktop OS - Linux rather than attempting a complete rewrite. Interesting multimedia related features from different research papers have been incorporated into Linux adding a new characteristic to it - QoS. It uses the HSFQ described in 4.1.4. Additionally, any slack time available after all the tasks are scheduled is dynamically shared among all nodes. One of their main goals was to provide backward compatibility. Applications that ran on Linux are able to run on QLinux with added benefits of these modifications. To achieve this using their hierarchical scheduler, they create a time-sharing class, to which, all legacy applications are assigned by default. This HSFQ is extended to the network layer also. The scheduling of packets is done based on a hierarchy of packet schedulers. This allows tasks with higher weights access to higher bandwidths.

Packet receiving is, however, implemented as a lazy receiver so that correct resource accounting can be done. When a packet is received, the task that is currently executing is not burdened with the overhead of handling the incoming packet. The task that needs the packet can read it during its allocated time. This can cause incorrect flow control interpretation by the sender. To avoid this, a kernel task is provided, to interpret the packets and send ACKs. All other forms of kernel overhead are also accounted into this task.

The disk scheduler used by QLinux is Cello. This is also hierarchical in design, consisting of two levels, one class independent and the other class dependent. Disk requests from class specific tasks are placed in respective queues. The scheduling algorithm takes the deadlines for each class into consideration and places them into a class independent FIFO, from where it performs the read/write. Over and above this, the scheduling algorithm takes rotational latency overhead into consideration when taking a decision, providing very good disk bandwidth.

The experimental results show the effectiveness of their system compared to Linux. The associated overheads are also very low - of the order of a few $\mu$ s. The Cello source code was apparently unstable to conduct any tests, so they have excluded it from any of their tests. This does not seem to be a major drawback as the Cello design looks robust and with a little tweaking it can definitely provide good performance. 
Apart from all the changes to the kernel, they have also provided some very powerful tools to dynamically modify the weights of the different applications. This provides the user complete flexibility in deciding the behavior of the tasks being run. This results in effective multiplexing of resources and service differentiation. Although they seem to have integrated a lot of the interesting mechanisms, they have not provided any functionality for admission control. Overall, their approach and results are very impressive.

\section{Conclusion}

The support provided by the general purpose OS's on the desktops do not provide sufficient support for multimedia, nor do special purpose OS's designed for only real-time applications. Hence, multimedia needs support in different areas to provide the intended viewing experience of the application. Due to the insufficient level of support, multimedia application developers are taking different routes. One of them is the deliberate reduction in content (RealAudio, QuickTime, MPEG-4 etc.) to provide viewable and deliverable speeds on desktops. This may be a good approach but I feel that, instead better support needs to be provided to match the high bandwidth and performance requirements of multimedia applications. Based on the above survey, I am convinced that the following feature set ought to be provided in an OS for multimedia applications:

- High level of user control in terms of preferences.

- Strict but flexible admission control.

- Simple and straightforward framework for resource allocation without too much system overhead.

- Fine grained resource accounting.

- Feedback path for smooth degradation of performance on overload.

- Support for multiple schedulers allowing a mix of applications to be run on the system.

- Minimal amount of backward compatibility for supporting legacy applications.

- Good distribution of slack time.

The introduction of Television sets revolutionized entertainment in the $20^{\text {th }}$ Century. I feel that multimedia will similarly revolutionize entertainment in the $21^{\text {st }}$ Century. As its name suggests, multimedia is the blending of various forms of media, providing a fertile area for further research aimed at supporting these applications on devices ranging from servers to desktops to handheld and embedded devices.

\section{Bibliography}

[1] T. Plagemann, V. Goebel, and P. Halvorsen, "Operating System Support for Multimedia Systems" Computer Communications Journal, Special Issue on Interactive Distributed Multimedia Systems and Telecommunications Services (IDMS '98), 1998.

[2] J. Nieh, J. G. Hanko, J. D. Northcutt, G. A. Wall, "SVR4 UNIX Scheduler Unacceptable for Multimedia Applications", Proceedings of the Fourth International Workshop on Network and OS Support for Digital Audio and Video, Nov' 93.

[3] Waldspurger, C. A. and Weihl, W. E., "Lottery scheduling: Flexible proportional share resource management", Proceedings of the First Symposium on OS Design and Implementation, Nov' 94

[4] J. Neih and M. S. Lam, "The design, implementation and evaluation of SMART: A scheduler for multimedia applications", Proceedings of ACM Symposium on OS Principles, St. Malo, France, October 1997.

[5] Miche Baker-Harvey, "ETI Resource Distributor: Guaranteed Resource Allocation and Scheduling in Multimedia Systems", Proceedings of the 3rd Symposium on Operating Systems Design and Implementation New Orleans, Feb' 99.

[6] Marc E. Fiuczynski, Richard P. Martin, Tsutomu Owa, Brian N. Bershad, "On Using Intelligent Network Interface Cards to Support Multimedia Application", 7th workshop on Network and OS Support for Digitial Audio Video July 1998, Cambridge, England.

[7] K. Jeffay, D. Stone, D. Poirier, "Yartos: Kernel Support for efficient, predictable real-time systems", Eighth IEEE Workshop on Real-Time OS and Software, May 1991.

[8] M. Jones, J. Barrera III, A. Forin, P. Leach, D. Rosu, and M. Rosu, "An overview of the Rialto Real-Time Architecture", Proceedings of the Seventh ACM SIGOPS European Workshop, September 1996.

[9] V. Sundaram, A. Chandra, P. Goyal, P. Shenoy, J. Sahni, and H. Vin, "Application Performance in the QLinux Multimedia Operating System," Proceedings of the Eighth ACM Conference on Multimedia, Los Angeles, CA, November 2000. 